\title{
Case Report: Myeloid Sarcoma Development During Treatment for B Cell Lymphoblastic Lymphoma in a Boy with KRAS/NRAS Gene Mutations
}

OncoTargets and Therapy

Xiuli Yuan ',*
Uet Yu (D',*
Senmin Chen'
Huanli Xu'
Meng Yi'
Xianping Jiang
Jianming Song'
Xiaowen Chen
Shiyang Chen
Zhenhu Lin
'
Changgang Li'
Feiqiu Wen'
Sixi Liu'
'Department of Hematology and
Oncology, Shenzhen Children's Hospital,
Shenzhen, People's Republic of China;
'Department of Pathology, Shenzhen
Children's Hospital, Shenzhen, People's
Republic of China; ${ }^{3}$ Institute for Medical
Research, Shenzhen Children's Hospital,
Shenzhen, People's Republic of China
*These authors contributed equally to
this work
this work

\begin{abstract}
Here, we report a rare case of a 12-year-old boy who was initially diagnosed with B cell lymphoblastic lymphoma (BLBL) and developed myeloid sarcoma (MS) eight months after chemotherapy. Next-generation sequencing (NGS) showed mutations of KRAS and NRAS genes in both the bone marrow and lymph node. He presented an abnormal karyotype of $46, \mathrm{XY},-9$, der $(16) \mathrm{t}(9 ; 16)$ (q13; q12), +mar. He received chemotherapy according to the South China Children's Leukemia Group 2016 protocol. Complete remission was achieved by the 15th day post-treatment. Eight months later and immediately prior to the start of maintenance therapy, the patient developed fever, skin nodules in both upper arms, and enlargement of bilateral testes. Pathological analysis of skin and testicular biopsies suggested the diagnosis of myeloid sarcoma (MS). Again, NGS examination showed mutations of KRAS and NRAS genes. The patient underwent haploidentical hematopoietic stem cell transplantation but unfortunately did not survive. The interval of eight-month interval between the initial disease onset and MS brings into question whether MS developed as part of the initial onset of disease or as a secondary tumor in association with chemotherapy. Thus, understanding the pathogenesis of MS involving abnormalities of lymphoid progenitors may assist in the prediction of prognosis and development of novel target therapies.
\end{abstract}

Keywords: B cell lymphoblastic lymphoma, myeloid sarcoma, KRAS, NRAS, case report

\section{Background}

Myeloid sarcoma (MS) is characterized as a myeloid neoplasm that mainly affects extramedullary sites, including the skin, lymph nodes, gastrointestinal tract, bone, soft tissue, and reproductive organs. ${ }^{1}$ Although MS affects all age groups of patients, the incidence of MS is low. MS is most often found in patients with newly or recently diagnosed acute myeloid leukemia (AML). The concurrence of MS with neoplasms derived from lymphoid progenitors has only been reported in few cases. ${ }^{2,3}$

Mutations of genes related to intracellular signaling pathways (such as the RAS/ RAF/MAPK and phosphoinositide-3 pathways) may alter the cell cycle via abnormal proliferation, differentiation, and apoptosis of cells. ${ }^{4}$ Among these, alterations of genes involved in the receptor tyrosine kinase (RTK)-RAS pathway, including NRAS and KRAS, are suspected to contribute significantly to the regulation and pathogenesis of MS. ${ }^{5,6}$ Patients who develop MS may have a poor prognosis. Therapies aimed at these genetic variations may help with the development of targeted strategies against MS.
Correspondence: Sixi Liu Department of Hematology and Oncology, Shenzhen Children's Hospital, Yitian Road No. 7019, Futian, Shenzhen, Guangdong 518038, People's Republic of China

Tel +86 189-3869-0206

Email tiger647@I26.com 


\section{Case Presentation}

A 12-year-old male patient with complaints of neck masses for a month and intermittent fever for two weeks was admitted to the Department of Hematology and Oncology at Shenzhen Children's Hospital, China. He was diagnosed with lymphadenitis at a local hospital a month before admission and was treated with intravenous ceftriaxone and oral amoxicillin, as well as intravenous methylprednisolone $(1.5 \mathrm{mg} / \mathrm{kg} /$ day $)$ for two days. The patient was previously healthy and denied any family history of malignant diseases.

Physical examination revealed multiple enlarged cervical and inguinal lymph nodes. The lymph nodes were moderate to firm in texture, with a maximum size of $2 \mathrm{~cm} \mathrm{x} 1 \mathrm{~cm}$, and mobile without adhesions to each other or neighboring tissues. The lymph nodes were slightly tender on palpation. Hepatosplenomegaly was noted. No other abnormalities were observed during the physical examination.

Laboratory evaluations revealed slight leukopenia with a white blood cell count of $4.37 \times 10^{9} / \mathrm{L}$ and a neutrophil count of $2.15 \times 10^{9} / \mathrm{L}$. Neither anemia nor thrombocytopenia was observed. The blood smear examination showed that blasts accounted for 5\% of total white blood cells. Elevated levels of alanine aminotransferase (ALT, $170 \mathrm{IU} /$ L), aspartate aminotransferase (AST, $175 \mathrm{IU} / \mathrm{L}$ ), and lactic dehydrogenase (LDH, $1007 \mathrm{IU} / \mathrm{L})$ were observed. All other laboratory findings (including direct and indirect bilirubin, creatinine, albumin, creatine kinase, and immunoglobulin levels) were within the normal range. Test results for antibodies to tuberculosis, Epstein-Barr virus, cytomegalovirus, Mycoplasma pneumoniae, and Chlamydia pneumoniae were negative.

Enhanced computed tomography (CT) scans of the neck and chest confirmed the presence of multiple enlarged cervical lymph nodes, particularly on the left side of the neck. No fluctuations or adhesions of the lymph nodes were observed. Hepatomegaly $(3 \mathrm{~cm}$ below the lowest right costal margin) and splenomegaly $(2.4 \mathrm{~cm}$ below the lowest left costal margin) were observed on ultrasound examination. No masses were observed in the abdomen or pelvis. Brain magnetic resonance imaging showed no abnormalities.

The bone marrow smear showed $15 \%$ of blast cells. These cells displayed a monotonous population of round and oval cells with scant cytoplasm. Vacuolated cytoplasm was observed in a few blasts. Approximately $1 \%$ of the blast cells showed basophilic staining with scant cytoplasm, folded nuclei, and absent nucleoli. Periodic acid-Schiff (PAS) staining was positive in most blasts, and peroxidase (POX) staining was positive in $43 \%$ of blast cells (Figure 1).

Flow cytometry analysis of bone marrow aspirates showed blasts accounting for $17.1 \%$ of the total cells. Blast cells were positive to CD19, CD10, HLA-DR, TdT, CD13 (partial), CD79a (partial), and myeloperoxidase (MPO; partial) (Figure 2). Expressions of CD34, CD33, CD117, CD20, CD15, CD3, CD56, and IgM were negative. Cytogenetic abnormalities with a karyotype of 46, XY, -9 , der (16) t (9; 16) (q13; q12), +mar were observed (Figure 3). Examinations of fusion genes for both acute lymphoblastic leukemia and myeloid leukemia were negative. Panel sequencing was performed to examine genes that related to lymphoid and myeloid neoplasms. Mutations of KRAS (c.38G>A, p.G13D) and NRAS (c.35G>A, p.G12D) genes were detected from the bone marrow sample. The variant allele fractions were $8.3 \%$ and $11.1 \%$, respectively.

A biopsy of the cervical lymph nodes on the left side of the neck was performed. Morphological examination

\section{Giemsa}

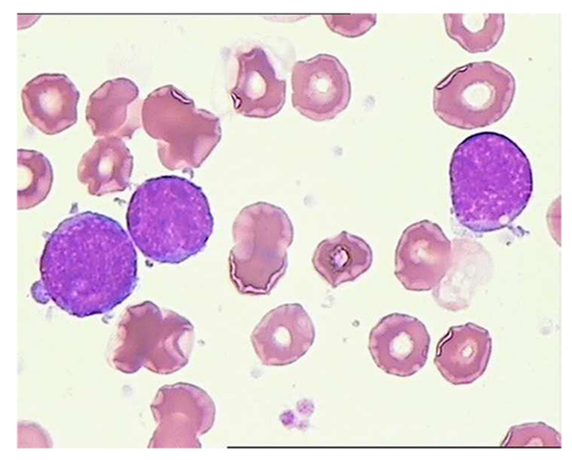

PAS

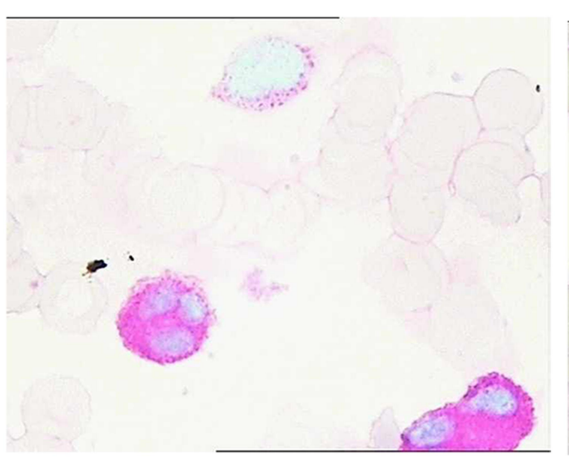

POX

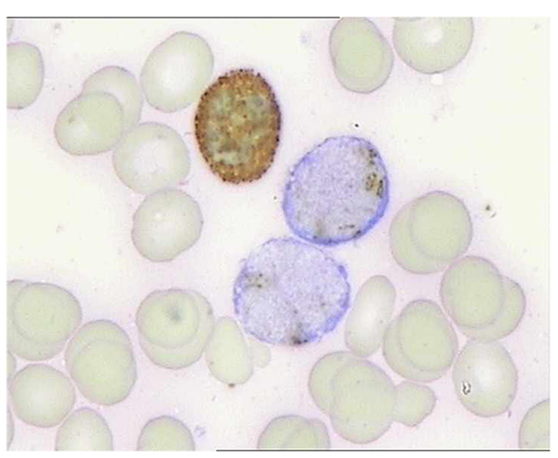

Figure I Morphological examination of the bone marrow showed cells that displayed a monotonous population of round and oval cells with scant cytoplasm by Giemsa staining. Peroxidase (POX) staining was positive in $43 \%$ of blast cells. Periodic acid-Schiff (PAS) staining was positive in most blasts. 

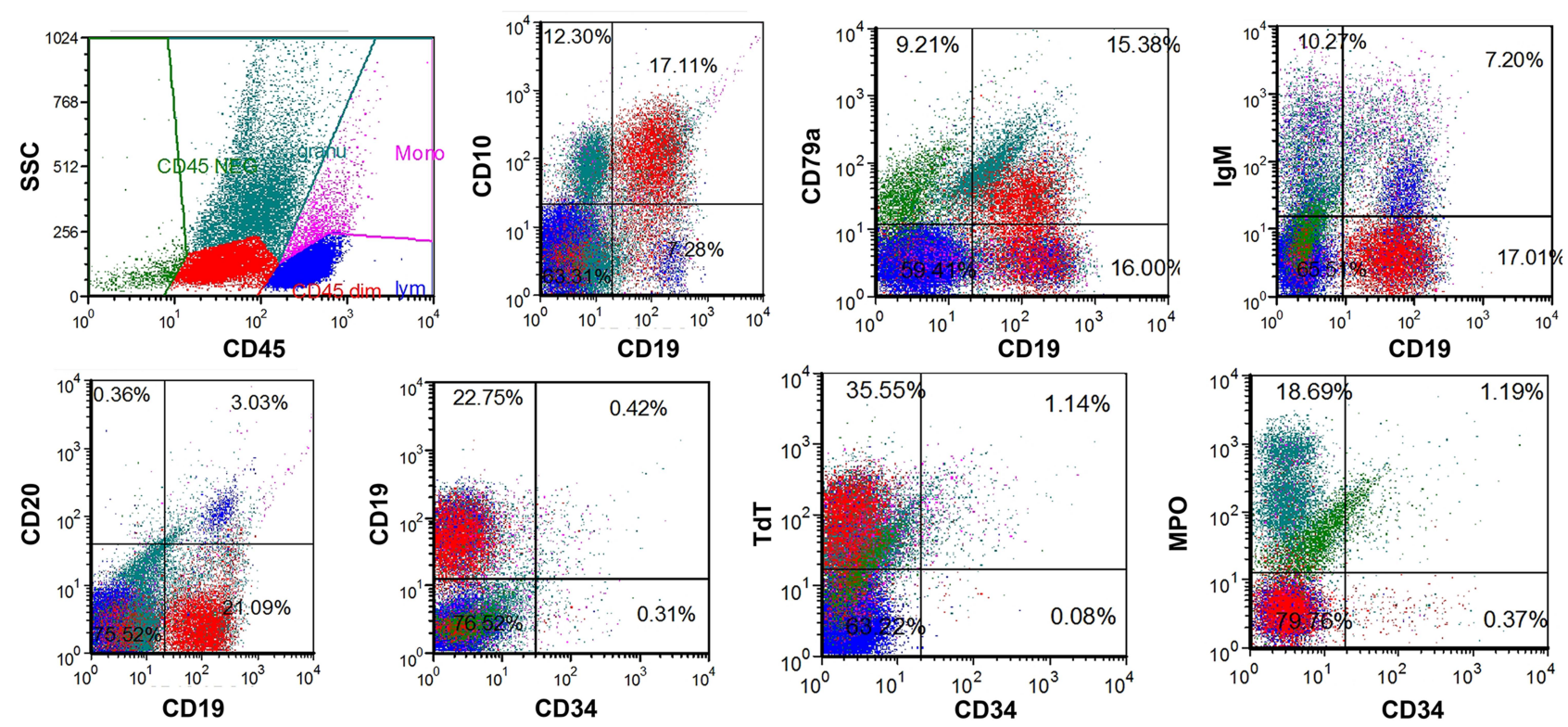

Figure 2 Flow cytometry plots of the bone marrow at the time of diagnosis showed blasts (CD45) that stained positive for CDI0, CDI9, CD79a, TdT, and MPO, and negative for CD34, CD20, IgM.

showed uniformly small to intermediate-sized cells. Immunohistochemistry (IHC) examination of the lymph node showed positive staining of CD10, CD79a, TdT, Ki-67 (>85\%), PAX5, CD1a (partial), CD20 (sporadic), $\mathrm{CD} 3$ (isolated), CD4 (few, isolated), CD7 (sporadic), CD8 (sporadic), CD99/MIC2, and CD2 (sporadic). MPO expression was observed (Figure 4). Expressions of ALK, CD30, and CD34 were negative. Next-generation sequencing (NGS) examination of the lymph node showed a mutation of the NRAS gene (c.35G>A, p.G12D). However, mutation of the KRAS gene was not detected in the lymph node. $\mathrm{B}$ cell receptor (BCR) rearrangement examination was positive. According to the $2016 \mathrm{WHO}$ classification, a diagnosis of BLBL was considered despite the expression of MPO. ${ }^{7}$ This patient was categorized as an intermediate-risk patient and was given chemotherapy according to the South China Children's Leukemia Group 2016 (SCCLG-2016) protocol.

Chemotherapy consisted of a prednisone experiment for seven days prior to induction therapy, an induction therapy of a cycle of VDLD (VCR/Dex/L-Peg-Asp /DNR) and two cycles of CAM+VL (CTX/Ara-C/6-MP $+\mathrm{VCR} /$ Preg-L-Asp), a consolidation therapy of four cycles of high dose MTX, and a reinduction therapy of a cycle of VDLD and a cycle of CAM+VL. Maintenance chemotherapy was prescribed with 6-MP+MTX+VD (VCR+Dex) and planned for a total period of 2.5 years. The patient achieved a good response to prednisone with a significant reduction of blasts in the peripheral blood on the 8th day of therapy. Bone marrow examination performed on the 15th day showed complete remission (CR) of the bone marrow by morphological examination. Flow cytometry of the bone marrow aspirate showed that the minimal residue disease was less than $0.01 \%$. The bone marrow of the patient remained in CR during the time of chemotherapy. No life-threatening infections or complications were observed.

Eight months after the initiation of chemotherapy and just prior to the start of maintenance therapy, the patient developed fever, skin nodules in both upper arms, and bilateral testicular enlargements. Both morphological and flow cytometry examination of the bone marrow performed at this time were negative. Biopsies of the skin and testes were performed. Histological examination of testicular tissues showed massive infiltration of tumor cells. IHC examination of the cells revealed positive staining for lysozyme, CD33, MPO, and Ki-67 (>95+), and negative staining for CD7, PAX-5, CD34, TdT. Histological examination of skin tissue showed massive proliferation of tumor cells. IHC of these cells revealed positive staining for lysozyme, MPO, CD33, Ki-67 (>95\% +), CD4 (mild), and negative staining for CD3, CD7, CD20, PAX-5, CD117, CD163, CD123, TdT, CD30, ALK. These results suggested MS of the skin and testes. NGS examinations of the skin and testicular samples showed mutations of both the KRAS (c.436G $>$ A, p. 


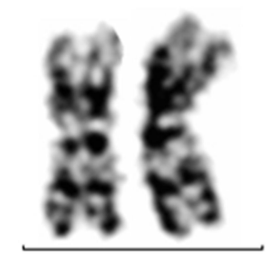

1

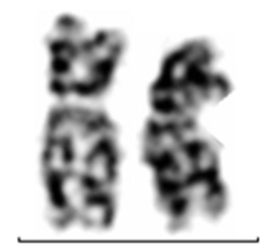

2

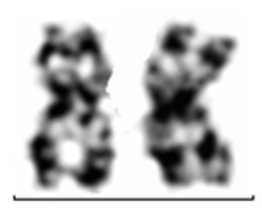

3

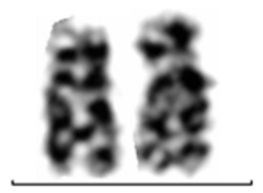

4

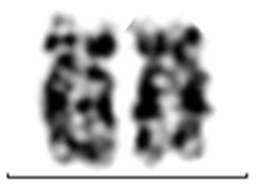

5

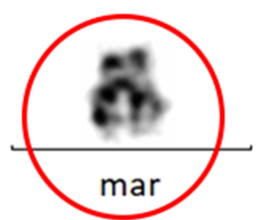

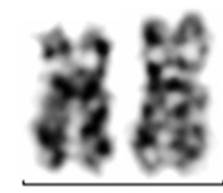

6

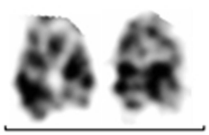

13

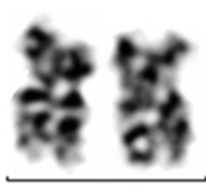

7

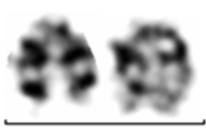

14

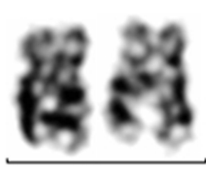

8

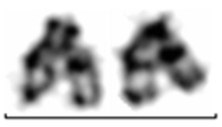

15

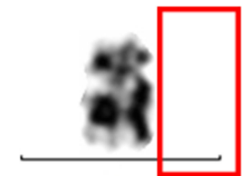

9

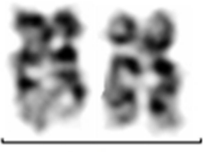

10

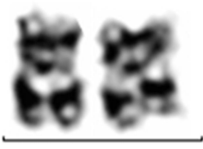

11
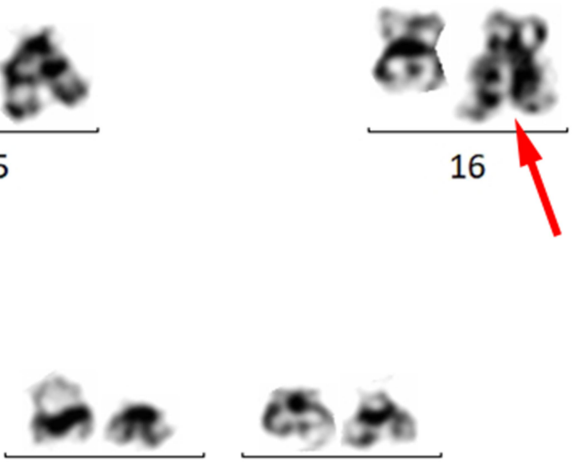

21

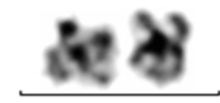

20

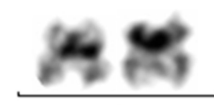

19

Figure 3 Cytogenetic examination of the bone marrow aspirates showed abnormal karyotype of $46, \mathrm{XY},-9$, der $(16) \mathrm{t}(9 ; \mathrm{I6})(\mathrm{q} \mid 3$; q $\mathbf{2})$, +mar. Analysis of chromosomes showed a deletion of chromosome 9 (red box), translocation of chromosome 9 and 16 (arrow) that formed a derivative chromosome (circle).

A146T) and NRAS genes (c.35G $>$ A, p.G12D). Positron emission CT examination showed nodular lesions with increased uptake of $18 \mathrm{~F}$-fluorodeoxyglucose (18F-FDG) in both testes. In addition, increased 18F-FDG uptakes were observed in the cervical vertebra, thoracic vertebra, and the right back ribs, suggesting metastasis to the bones.

The patient was subsequently given high-intensity chemotherapy consisting of etoposide, Ara-C, and DNR. After two cycles of chemotherapy, he received haploidentical hematopoietic stem cell transplantation. His mother served as the donor. Unfortunately, this patient did not survive due to severe graft-versus-host disease and other complications following the transplant.

\section{Discussion}

MS is commonly seen in conjunction with, or during relapses of AML, myeloproliferative neoplasm, and myelodysplastic syndrome. MS may be observed in up to $40 \%$ of children with AML. ${ }^{1,8,9}$ However, the incidence of MS remains low within the general oncology pediatric population. MS complicated with acute lymphoblastic leukemia or lymphoma only has been identified rare cases, ${ }^{2,3,10,11}$ most of which were initially misdiagnosed as nonHodgkin's lymphoma. Thus, extensive evaluations were required to overcome the challenges in diagnosing MS complicated with other diseases, especially among those without bone marrow involvement.

In the present case, the key presenting abnormalities of the patient were the enlarged cervical and inguinal lymph nodes. Masses were not observed at other sites. Blasts comprised $20 \%$ of the total bone marrow cells and expressed markers for B cell blasts, including CD19, CD79a, and PAX5. The patient's lymph node biopsy results also featured typical histological characteristics of lymphoblastic lymphoma, including the expression of CD10, CD79a, and TdT. An unusual finding of this case 

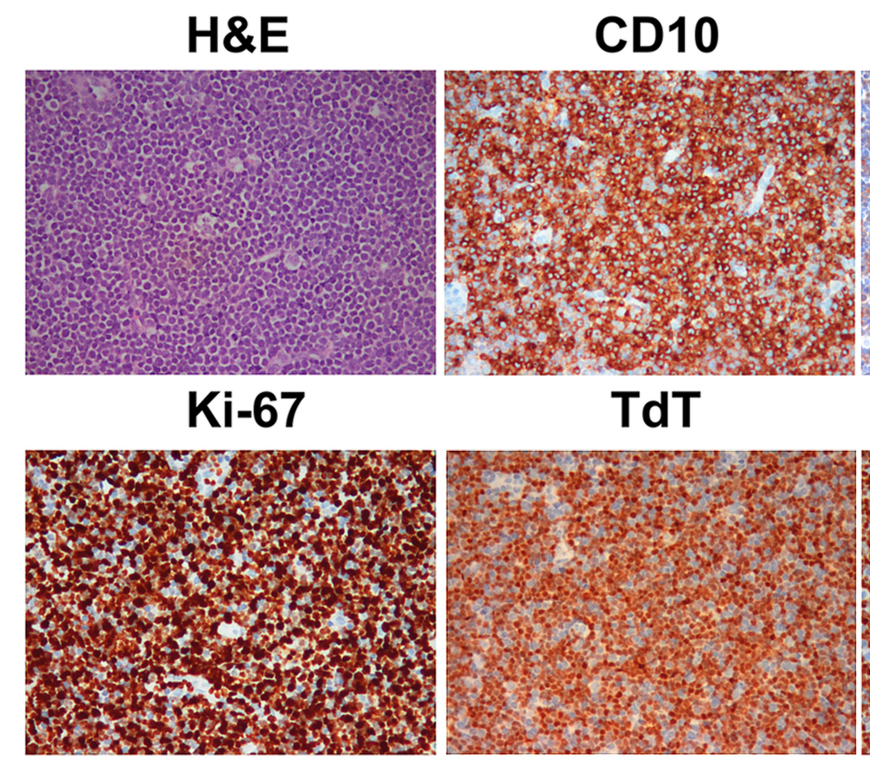

CD79a

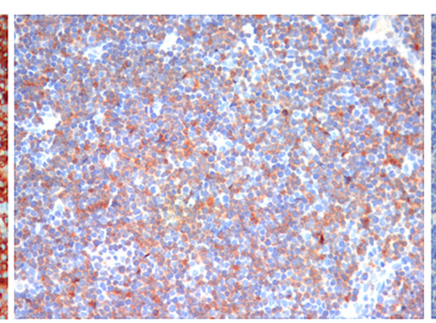

PAX-5

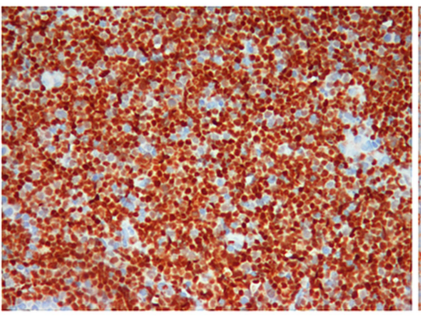

CD20

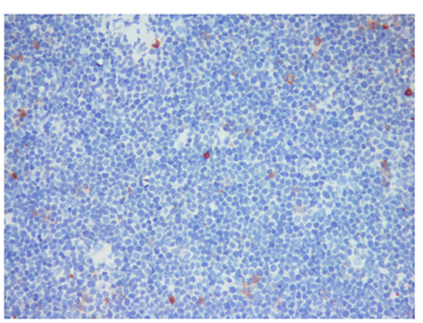

MPO

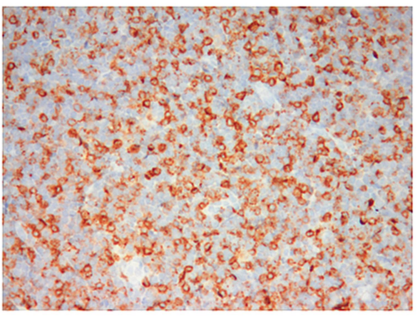

Figure 4 Histological examination of the cervical lymph node biopsy of the left neck showed uniformly small to intermediate-sized cells by hematoxylin and eosin ( $\mathrm{H} \& \mathrm{E}$ ) staining. Immunohistochemistry examination showed strong positive staining for CDI0, CD79a, TdT, Ki-67 (>85\%), PAX5, and myeloperoxidase, and partial staining of CD20.

was the strong reaction of MPO, a typical myeloid marker identified in the lymph node by immunohistochemistry. MPO was also detected at low level in the blasts by flow cytometry. Another pan-myeloid marker, CD13, was partially demonstrated in the bone marrow by flow cytometry (not shown). However, this is a pan-myeloid marker commonly seen in B-ALL, especially for B-ALL that involves the translocation of chromosomes 9 and 22 and fusion of BCR/ABL1 genes. ${ }^{12,13}$ Other myeloid associated markers including CD15 and CD117 were not expressed. Expression of monocytic markers including CD14 and CD64 was not observed. In fact, MPO expression could be observed in up to $22 \%$ of pediatric BALL patients. ${ }^{14}$ Another concern in the diagnosis of this patient was the use of methylprednisolone before admission that might have lysed part of the blasts. Thus, the percentage of blasts in the bone marrow (15\%) could have been underestimated hence suggestive of a diagnosis of mixed phenotypic acute leukemia (MPAL) according to the 2016 WHO guideline. However, based on the existing evaluations of the bone marrow and lymph nodes, a diagnosis of BLBL with MPO expression was considered for this patient.

The cause of MS in this patient remains uncertain. An important aspect of this case was the mutation of both the NRAS and KRAS genes. Concurrent mutations of both NRAS and KRAS gene have rarely been reported because these mutations are often mutually exclusive. This is because additional mutations of the RAS genes would not escalate the discomposed RAS signaling pathway. ${ }^{15}$ However, the presence of both NRAS and KRAS mutations has been previously identified in few pediatric BALL patients. ${ }^{16}$ In this case, both NRAS and KRAS mutations were detected at initial diagnosis and at the development of MS. Moreover, NRAS and KRAS genes participate as part of the receptor tyrosine kinase (RTK)-RAS pathway. ${ }^{5,17}$ Recent genomic studies reveal that mutations associated with the RTK-RAS pathway are frequently observed in pediatric patients with B-ALL and are probably more prevalent in the Chinese population. ${ }^{15,16,18}$ Although mutations of RAS genes have been shown associated with poorer outcomes in pediatric ALL, AML, MPAL, and infant leukemia patients, the association of RAS mutations and patient prognosis remains undefined, warranting larger cohort studies. ${ }^{15,19}$

In this case, MS was identified approximately eight months after the initiation of chemotherapy. A plausible explanation is that MS developing at extramedullary sites requires more oncogenetic mutations. ${ }^{9}$ The high-intensity chemotherapy given to this patient may have accelerated the mutations that led to the development of MS as a second tumor. $^{20-24}$ This patient had the abnormal chromosomal karyotype of $46, \mathrm{XY},-9$, der $(16) \mathrm{t}(9 ; 16)$ (q13; q12), +mar. However, no evidence suggests that this chromosomal abnormality contributes to an increased susceptibility to oncogenic clonal evolution. Whether these chromosomal abnormalities increased the risk for the development of secondary tumors requires further investigation. 
Another hypothesis of the cause of MS was the crosslineage transdifferentiation of B cell progenitors in BLBL to MS. In adults, transdifferentiations of BALL or other lymphoid progenitors into neoplasms of other lineage such as histiocytic sarcoma/leukemia have been reported in few cases. $^{25-28}$ Transdifferentiations between neoplasms of tumor cells from other lineages have also been reported in sporadic adult cases. ${ }^{29,30}$ Most of these patients feature chromosomal and genetic abnormalities including the mutation of RAS genes. The presence of NRAS and KRAS genes and the expression of MPO may suggest the potential of transdifferentiation of B cells into myeloid blasts. However, transdifferentiation of BLBL to MS has not been reported in pediatric patients and needs further investigations.

\section{Conclusions}

In conclusion, we report a boy who developed MS after chemotherapy for BLBL after a period of approximately eight months. The exact mechanism of the development of MS in this patient remains uncertain. A diagnosis of BLBL was considered despite the expression of myeloid marker MPO. This patient exhibited certain cytogenetic abnormalities, but fusion genes were not presented. NGS evaluations revealed mutations of both NRAS and KRAS genes that may contribute to the genetic origin of MS. Therefore, understanding the mechanisms of the development of MS in this patient may help with the prediction of prognosis and the creation and advancement of therapeutic strategies for targeted treatments, and prevention of secondary malignancies.

\section{Abbreviations}

6-MP, 6-mercaptopurine; ALL, acute lymphoblastic leukemia; AML, acute myeloid leukemia; Ara-C, cytosine arabinoside; BLBL, B cell lymphoblastic lymphoma; CTX, cyclophosphamide; Dex, dexamethasone; DNR, daunoblastina; L-Peg-Asp, L-asparaginase; MPO, myeloperoxidase; MS, myeloid sarcoma; MTX, methotrexate; VCR, vincristine.

\section{Data Sharing Statement}

Not applicable.

\section{Ethical Approval and Informed Consent}

Ethical approval is not required for this Case report. Written informed consent was obtained from the patient's parent.

\section{Consent for Publication}

Written informed consent was obtained from the patient's parent for publication of this paper and any accompanying images.

\section{Acknowledgments}

We thank A/Prof Ronald Grant from the Hospital for Sick Children in Toronto, Canada for the long-time support to help us to provide better care for our pediatric oncology patients. Xiuli Yuan and Uet Yu are co-first authors for this study.

\section{Author Contributions}

All authors made a significant contribution to the work reported, whether that is in the conception, study design, execution, acquisition of data, analysis and interpretation, or in all these areas; took part in drafting, revising or critically reviewing the article; gave final approval of the version to be published; have agreed on the journal to which the article has been submitted; and agree to be accountable for all aspects of the work.

\section{Funding}

Sanming Project of Medicine in Shenzhen (SZSM201512033), Shenzhen Fund for Guangdong Provincial High-level Clinical Key Specialties (SZGSP012), and Shenzhen Key Medical Discipline Construction Fund (SZXK034) supported the genetic assessment and data analysis for this paper.

\section{Disclosure}

The authors have no competing interests to declare.

\section{References}

1. Magdy M, Abdel Karim N, Eldessouki I, Gaber O, Rahouma M, Ghareeb M. Myeloid Sarcoma. Oncol Res Treat. 2019;42(4):22 4-229. doi:10.1159/000497210

2. Khanna G, Damle NA, Agarwal S, et al. Mixed phenotypic acute leukemia (mixed myeloid/b-cell) with myeloid sarcoma of the thyroid gland: a rare entity with rarer association - detected on FDG PET/CT. Indian J Nucl Med. 2017;32(1):46-49. doi:10.4103/0972-3919.198478

3. Takakuwa $T$, Nakane $T$, Ohsawa $M$, et al. The first case of non-leukemic sarcoma composed of mixed-phenotype acute leukemia, B/ myeloid, not otherwise specified. Intern Med. 2018;57 (8):1155-1158. doi:10.2169/internalmedicine.9144-17

4. de Ridder JAM, Knijn N, Wiering B, de Wilt JHW, Nagtegaal ID. Lymphatic invasion is an independent adverse prognostic factor in patients with colorectal liver metastasis. Ann Surg Oncol. 2015;22 (Suppl S3):S638-S645. doi:10.1245/s10434-015-4562-8

5. Choi M, Jeon YK, Sun C-H, et al. RTK-RAS pathway mutation is enriched in myeloid sarcoma. Blood Cancer J. 2018;8(5):43. doi:10.1038/s41408-018-0083-6 
6. Choi SM, Andea AA, Wang M, et al. KRAS mutation in secondary malignant histiocytosis arising from low grade follicular lymphoma. Diagn Pathol. 2018;13(1):78. doi:10.1186/s13000-018-0758-0

7. Arber DA, Orazi A, Hasserjian R, et al. The 2016 revision to the World Health Organization classification of myeloid neoplasms and acute leukemia. Blood. 2016;127(20):2391-2405. doi:10.1182/blood2016-03-643544

8. Støve HK, Sandahl JD, Abrahamsson J, et al. Extramedullary leukemia in children with acute myeloid leukemia: a population-based cohort study from the Nordic Society of Pediatric Hematology and Oncology (NOPHO). Pediatr Blood Cancer. 2017;64(12):e26520. doi: $10.1002 /$ pbc. 26520

9. Zhou T, Bloomquist MS, Ferguson LS, et al. Pediatric myeloid sarcoma: a single institution clinicopathologic and molecular analysis. Pediatr Hematol Oncol. 2020;37(1):76-89. doi:10.1080/ 08880018.2019.1683107

10. Wang Y, Wen S, Niu Z, Xing L, Wang F, Zhang X. T cell acute lymphoblastic lymphoma complicated with myeloid sarcoma in an adult: a case report. Oncol Lett. 2017;14(6):8178-8182. doi:10.3892/ ol.2017.7187

11. Seifert RP, Bulkeley W, Zhang L, Menes M, Bui MM. A practical approach to diagnose soft tissue myeloid sarcoma preceding or coinciding with acute myeloid leukemia. Ann Diagn Pathol. 2014;18 (4):253-260. doi:10.1016/j.anndiagpath.2014.06.001

12. Dalal BI, Al Mugairi A, Pi S, et al. Aberrant expression of CD13 identifies a subgroup of standard-risk adult acute lymphoblastic leukemia with inferior survival. Clin Lymphoma Myeloma Leuk. 2014;14(3):239-244. doi:10.1016/j.clml.2013.10.003

13. Knez VM, Carstens BJ, Swisshelm KL, McGranahan AN, Liang X. Heterogeneity of abnormal RUNX1 leading to clinicopathologic variations in childhood b-lymphoblastic leukemia. Am J Clin Pathol. 2015;144(2):305-314. doi:10.1309/AJCPVY5E5OMMYBFJ

14. McGinnis E, Yang D, Au N, et al. Clinical and laboratory features associated with myeloperoxidase expression in pediatric B-lymphoblastic leukemia. Cytometry B Clin Cytom. 2020. doi:10.1002/cyto.b.21966

15. Liang DC, Chen SH, Liu HC, et al. Mutational status of NRAS, KRAS, and PTPN11 genes is associated with genetic/cytogenetic features in children with B-precursor acute lymphoblastic leukemia. Pediatr Blood Cancer. 2018;65(2):e26786. doi:10.1002/pbc.26786

16. Zhang H, Wang H, Qian X, et al. Genetic mutational analysis of pediatric acute lymphoblastic leukemia from a single center in China using exon sequencing. BMC Cancer. 2020;20(1):211. doi:10.1186/ s12885-020-6709-7

17. Arnold MA, Barr FG. Molecular diagnostics in the management of rhabdomyosarcoma. Expert Rev Mol Diagn. 2017;17(2):189-194. doi:10.1080/14737159.2017.1275965
18. Li JF, Dai YT, Lilljebjörn H, et al. Transcriptional landscape of B cell precursor acute lymphoblastic leukemia based on an international study of 1223 cases. Proc Natl Acad Sci U S A. 2018;115(50): E11711-e11720. doi:10.1073/pnas.1814397115

19. Driessen EM, van Roon EH, Spijkers-Hagelstein JA, et al. Frequencies and prognostic impact of RAS mutations in MLL-rearranged acute lymphoblastic leukemia in infants. Haematologica. 2013;98 (6):937-944. doi:10.3324/haematol.2012.067983

20. Bhatia S. Therapy-related myelodysplasia and acute myeloid leukemia. Semin Oncol. 2013;40(6):666-675. doi:10.1053/j. seminoncol.2013.09.013

21. Chua CC, Fleming S, Wei AH. Clinicopathological aspects of therapy-related acute myeloid leukemia and myelodysplastic syndrome. Best Pract Res Clin Haematol. 2019;32(1):3-12. doi:10.1016/j.beha.2019.02.007

22. Godley LA, Larson RA. Therapy-related myeloid leukemia. Semin Oncol. 2008;35(4):418-429. doi:10.1053/j.seminoncol.2008.04.012

23. Larson RA. Therapy-related myeloid neoplasms. Haematologica. 2009;94(4):454-459. doi:10.3324/haematol.2008.005157

24. Qing X, Panosyan E, Yue C, et al. Therapy-related myeloid neoplasm in an 18-year-old boy with B-lymphoblastic leukemia. Exp Mol Pathol. 2017;103(3):263-266. doi:10.1016/j.yexmp.2017.11.007

25. Fraser CR, Wang W, Gomez M, et al. Transformation of chronic lymphocytic leukemia/small lymphocytic lymphoma to interdigitating dendritic cell sarcoma: evidence for transdifferentiation of the lymphoma clone. Am J Clin Pathol. 2009;132(6):928-939. doi:10.1309/ajcpwq0i0dgxbmho

26. Ansari J, Naqash AR, Munker R, et al. Histiocytic sarcoma as a secondary malignancy: pathobiology, diagnosis, and treatment. Eur J Haematol. 2016;97(1):9-16. doi:10.1111/ejh.12755

27. Thakral B, Khoury JD. Histiocytic sarcoma: secondary neoplasm or "transdifferentiation" in the setting of B-acute lymphoblastic leukemia. Blood. 2016;128(20):2475. doi:10.1182/blood-2016-08-735795

28. Rassidakis GZ, Stromberg O, Xagoraris I, Jatta K, Sonnevi K. Trametinib and Dabrafenib in histiocytic sarcoma transdifferentiated from chronic lymphocytic leukemia with a K-RAS and a unique BRAF mutation. Ann Hematol. 2020;99(3):649-651. doi:10.1007/ s00277-020-03941-7

29. Steussy B, Lekostaj J, Qian Q, et al. Leukemic transdifferentiation of follicular lymphoma into an acute histiocytic leukemia in a 52-yearold caucasian woman. Lab Med. 2016;47(2):155-157. doi:10.1093/ labmed/lmw011

30. Rupp NJ, Rechsteiner M, Freiberger SN, et al. New observations in tumor cell plasticity: mutational profiling in a case of metastatic melanoma with biphasic sarcomatoid transdifferentiation. Virchows Arch. 2018;473(4):517-521. doi:10.1007/s00428-018-2376-3
OncoTargets and Therapy

\section{Publish your work in this journal}

OncoTargets and Therapy is an international, peer-reviewed, open access journal focusing on the pathological basis of all cancers, potential targets for therapy and treatment protocols employed to improve the management of cancer patients. The journal also focuses on the impact of management programs and new therapeutic

Submit your manuscript here: https://www.dovepress.com/oncotargets-and-therapy-journal agents and protocols on patient perspectives such as quality of life, adherence and satisfaction. The manuscript management system is completely online and includes a very quick and fair peer-review system, which is all easy to use. Visit http://www.dovepress.com/ testimonials.php to read real quotes from published authors. 